\title{
The Euclidean Onofri inequality in higher dimensions
}

\author{
Manuel del Pino ${ }^{1}$ and Jean Dolbeault ${ }^{2}$ \\ ${ }^{1}$ Departamento de Ingeniería Matemática and CMM, UMI CNRS nr. 2807, Universidad de Chile, \\ Casilla 170 Correo 3, Santiago, Chile, E-mail: delpino@dim.uchile.cl, \\ ${ }^{2}$ Ceremade, UMR CNRS nr. 7534, Université Paris-Dauphine, Place de Lattre de Tassigny, 75775 \\ Paris Cedex 16, France, E-mail: dolbeaul@ceremade.dauphine.fr.
}

Correspondence to be sent to: delpino@dim.uchile.cl

The classical Onofri inequality in the two-dimensional sphere assumes a natural form in the plane when transformed via stereographic projection. We establish an optimal version of a generalization of this inequality in the $d$-dimensional Euclidean space for any $d \geq 2$, by considering the endpoint of a family of optimal Gagliardo-Nirenberg interpolation inequalities. Unlike the two-dimensional case, this extension involves a rather unexpected Sobolev-Orlicz norm, as well as a probability measure no longer related to stereographic projection.

Keywords: Sobolev inequality; logarithmic Sobolev inequality; Onofri inequalities; Gagliardo-Nirenberg inequalities; interpolation; extremal functions; optimal constants; stereographic projection

Mathematics Subject Classification (2010): 26D10; 46E35; 58E35

\section{Introduction and main result}

The Onofri inequality as stated in Onofri [1982] asserts that

$$
\log \left(\int_{\mathbb{S}^{2}} e^{v} d \sigma_{2}\right)-\int_{\mathbb{S}^{2}} v d \sigma_{2} \leq \frac{1}{4}\|\nabla v\|_{\mathrm{L}^{2}\left(\mathbb{S}^{2}, d \sigma_{2}\right)}^{2}
$$

for any function $v \in \mathrm{H}^{1}\left(\mathbb{S}^{2}, d \sigma_{2}\right)$. Here $d \sigma_{2}$ denotes the standard surface measure on the two-dimensional unit sphere $\mathbb{S}^{2} \subset \mathbb{R}^{3}$, up to a normalization factor $\frac{1}{4 \pi}$ so that $\int_{\mathbb{S}^{2}} 1 d \sigma_{2}=1$.

Using stereographic projection from $\mathbb{S}^{2}$ onto $\mathbb{R}^{2}$, that is defining $u$ by

$$
u(x)=v(y) \quad \text { with } \quad y=\left(y_{1}, y_{2}, y_{3}\right), \quad y_{1}=\frac{2 x_{1}}{1+|x|^{2}}, \quad y_{2}=\frac{2 x_{2}}{1+|x|^{2}}, \quad y_{3}=\frac{1-|x|^{2}}{1+|x|^{2}}
$$

for any $x=\left(x_{1}, x_{2}\right) \in \mathbb{R}^{2}$, then (1) can be reformulated into the Euclidean Onofri inequality, namely

$$
\log \left(\int_{\mathbb{R}^{2}} e^{u} d \mu_{2}\right)-\int_{\mathbb{R}^{2}} u d \mu_{2} \leq \frac{1}{16 \pi}\|\nabla u\|_{L^{2}\left(\mathbb{R}^{2}, d x\right)}^{2}
$$

for any $u \in \mathrm{L}^{1}\left(\mathbb{R}^{2}, d \mu_{2}\right)$ such that $\nabla u \in \mathrm{L}^{2}\left(\mathbb{R}^{2}, d x\right)$, where

$$
d \mu_{2}(x):=\frac{d x}{\pi\left(1+|x|^{2}\right)^{2}}
$$

is again a probability measure.

The purpose of this note is to obtain an (optimal) extension of inequality (2) to any space dimension. There is a vast literature on Onofri's inequality, and we shall only mention a few works relevant to our main result

November 20, 2018

(C) 2012 by the authors. This paper may be reproduced, in its entirety, for non-commercial purposes. 
below. Onofri's inequality with a non-optimal constant was first established by J. Moser in Moser [1970/71], a work prior to that of E. Onofri, Onofri [1982]. For this reason, the inequality is sometimes called the MoserOnofri inequality. We also point out that Onofri's paper is based on an earlier result of T. Aubin, Aubin [1979]. We refer the interested reader to Ghigi 2005] for a recent account on the Moser-Onofri inequality. The inequality has an interesting version in the cylinder $\mathbb{R} \times \mathbb{S}^{1}$, see Dolbeault et al. [2008], which is however out of the scope of the present work.

In this note, we will establish that the Euclidean version of Onofri's inequality (2) can be extended to an arbitrary dimension $d \geq 3$ in the following manner. Let us consider the probability measure

$$
d \mu_{d}(x):=\frac{d}{\left|\mathbb{S}^{d-1}\right|} \frac{d x}{\left(1+|x|^{\frac{d}{d-1}}\right)^{d}} .
$$

Let us denote

$$
\mathrm{R}_{d}(X, Y):=|X+Y|^{d}-|X|^{d}-d|X|^{d-2} X \cdot Y, \quad(X, Y) \in \mathbb{R}^{d} \times \mathbb{R}^{d},
$$

which is a polynomial if $d$ is even. We define

$$
\mathrm{H}_{d}(x, p):=\mathrm{R}_{d}\left(-\frac{d|x|^{-\frac{d-2}{d-1}}}{1+|x|^{\frac{d}{d-1}}} x, \frac{d-1}{d} p\right), \quad(x, p) \in \mathbb{R}^{d} \times \mathbb{R}^{d},
$$

and

$$
\mathcal{Q}_{d}[u]:=\frac{\int_{\mathbb{R}^{d}} \mathrm{H}_{d}(x, \nabla u) d x}{\log \left(\int_{\mathbb{R}^{d}} e^{u} d \mu_{d}\right)-\int_{\mathbb{R}^{d}} u d \mu_{d}} .
$$

The following is our main result.

Theorem 1.1. With the above notation, for any smooth compactly supported function u, we have

$$
\log \left(\int_{\mathbb{R}^{d}} e^{u} d \mu_{d}\right)-\int_{\mathbb{R}^{d}} u d \mu_{d} \leq \alpha_{d} \int_{\mathbb{R}^{d}} \mathrm{H}_{d}(x, \nabla u) d x .
$$

The optimal constant $\alpha_{d}$ is explicit and given by

$$
\alpha_{d}=\frac{d^{1-d} \Gamma(d / 2)}{2(d-1) \pi^{d / 2}} .
$$

Small multiples of the function

$$
v(x)=-d \frac{x \cdot \mathrm{e}}{|x|^{\frac{d-2}{d-1}}\left(1+|x|^{\frac{d}{d-1}}\right)}
$$

for a unit vector e are approximate extremals of (3) in the sense that

$$
\lim _{\varepsilon \rightarrow 0} \mathcal{Q}_{d}[\varepsilon v]=\frac{1}{\alpha_{d}}
$$

A rather unexpected feature of inequality (3) when compared with Onofri's inequality (2), is that it involves an inhomogeneous Sobolev-Orlicz type norm. As we will see below, as a by-product of the proof we obtain a new Poincaré inequality in entire space, (7) below, of which the function $v$ defined by (4) is an extremal.

Example 1.2. If $d=2, \int_{\mathbb{R}^{d}} \mathrm{H}_{2}(x, \nabla u) d x=\frac{1}{4} \int_{\mathbb{R}^{2}}|\nabla u|^{2} d x$ and we recover Onofri's inequality (3) as in Dolbeault [2011], with optimal constant $1 / \alpha_{2}=4 \pi$. On the other hand, if for instance $d=4$, we find that $H_{4}(x, \nabla u)$ is a fourth order polynomial in the partial derivatives of $u$, since $\mathrm{R}_{4}(X, Y)=4(X \cdot Y)^{2}+|Y|^{2}\left(|Y|^{2}+4 X \cdot Y+\right.$ $\left.2|X|^{2}\right)$.

Extensions of inequality (2) to higher dimensions were already obtained long ago. Inequality (1) was generalized to the $d$-dimensional sphere in Beckner [1993], Carlen and Loss [1992], where natural conformally invariant, non-local generalizations of the Laplacian were used. Those operators are of different nature than the ones in Theorem 1.1. Indeed, no clear connection through, for instance, stereographic projection is present. See also Kim [2000], Kawohl and Lucia [2008] in which bounded domains are considered. 
Inequality (3) determines a natural Sobolev space in which it holds. Indeed, a classical completion argument with respect to a norm corresponding to the integrals defined in both sides of the inequality determines a space on which the inequality still holds. This space can be identified with the set of all functions $u \in \mathrm{L}^{1}\left(\mathbb{R}^{d}, d \mu_{d}\right)$ such that the distribution $\nabla u$ is a square integrable function. To avoid technicalities, computations will only be done for smooth, compactly supported functions.

Our strategy is to consider the Euclidean inequality of Theorem 1.1 as the endpoint of a family of optimal interpolation inequalities discovered in Del Pino and Dolbeault [2002b] and then extended in Del Pino and Dolbeault 2002a]. These inequalities can be stated as follows.

Theorem 1.3. Let $p \in(1, d], a>1$ such that $a \leq \frac{p(d-1)}{d-p}$ if $p<d$, and $b=p \frac{a-1}{p-1}$. There exists a positive constant $\mathrm{C}_{p, a}$ such that, for any function $f \in \mathrm{L}^{a}\left(\mathbb{R}^{d}, d x\right)$ with $\nabla f \in \mathrm{L}^{p}\left(\mathbb{R}^{d}, d x\right)$, we have

$$
\|f\|_{\mathrm{L}^{b}\left(\mathbb{R}^{d}\right)} \leq \mathrm{C}_{p, a}\|\nabla f\|_{\mathrm{L}^{p}\left(\mathbb{R}^{d}\right)}^{\theta}\|f\|_{\mathrm{L}^{a}\left(\mathbb{R}^{d}\right)}^{1-\theta} \quad \text { with } \theta=\frac{(a-p) d}{(a-1)(d p-(d-p) a)}
$$

if $a>p$. A similar inequality also holds if $a<p$, namely

$$
\|f\|_{\mathrm{L}^{a}\left(\mathbb{R}^{d}\right)} \leq \mathrm{C}_{p, a}\|\nabla f\|_{\mathrm{L}^{p}\left(\mathbb{R}^{d}\right)}^{\theta}\|f\|_{\mathrm{L}^{b}\left(\mathbb{R}^{d}\right)}^{1-\theta} \quad \text { with } \theta=\frac{(p-a) d}{a(d(p-a)+p(a-1))} .
$$

In both cases, equality holds for any function taking the form

$$
f(x)=A\left(1+B\left|x-x_{0}\right|^{\frac{p}{p-1}}\right)_{+}^{-\frac{p-1}{a-p}} \quad \forall x \in \mathbb{R}^{d}
$$

for some $\left(A, B, x_{0}\right) \in \mathbb{R} \times \mathbb{R} \times \mathbb{R}^{d}$, where $B$ has the sign of $a-p$.

While in Del Pino and Dolbeault 2002a], only the case $p<d$ was considered, the proof there actually applies to also cover the case $p=d$, for any $a \in(1, \infty)$.

For $a=p$, inequality (5) degenerates into an equality. By substracting it to the inequality, dividing by $a-p$ and taking the limit as $a \rightarrow p_{+}$, we obtain an optimal Euclidean $\mathrm{L}^{p}$-Sobolev logarithmic inequality which goes as follows. Assume that $1<p \leq d$. Then for any $u \in \mathrm{W}^{1, p}\left(\mathbb{R}^{d}\right)$ with $\int_{\mathbb{R}^{d}}|u|^{p} d x=1$ we have

$$
\int_{\mathbb{R}^{d}}|u|^{p} \log |u|^{p} d x \leq \frac{d}{p} \log \left[\beta_{p, d} \int_{\mathbb{R}^{d}}|\nabla u|^{p} d x\right], \quad \text { where } \quad \beta_{p, d}:=\frac{p}{d}\left(\frac{p-1}{e}\right)^{p-1} \frac{1}{\pi^{\frac{p}{2}}}\left[\frac{\Gamma\left(\frac{d}{2}+1\right)}{\Gamma\left(d \frac{p-1}{p}+1\right)}\right]^{\frac{p}{d}}
$$

is the optimal constant. Equality holds if and only if for some $\sigma>0$ and $x_{0} \in \mathbb{R}^{d}$

$$
u(x)=\left[\frac{1}{2 \pi^{\frac{d}{2}}} \frac{p}{p-1} \frac{\Gamma\left(\frac{d}{2}\right)}{\Gamma\left(d \frac{p-1}{p}\right)}\left(\frac{p}{\sigma}\right)^{d \frac{p-1}{p}}\right]^{\frac{1}{p}} e^{-\frac{1}{\sigma}\left|x-x_{0}\right|^{\frac{p}{p-1}}} \quad \forall x \in \mathbb{R}^{d} .
$$

This inequality has been established in Del Pino and Dolbeault 2003] when $p<d$ and in general in Gentil 2003]; see also Cordero-Erausquin et al. [2004], Del Pino et al. [2004].

When $p<d$, the endpoint $a=\frac{p(d-1)}{d-p}$ corresponds to the usual optimal Sobolev inequality, for which the extremal functions were already known from the celebrated papers by T. Aubin and G. Talenti, Aubin [1976], Talenti [1976]. See also Bliss [1930], Rosen [1971] for earlier related computations, which provided the value of some of the best constants.

When $p=d$, Theorem 1.1 will also be obtained by passing to a limit, namely as $a \rightarrow+\infty$. In this way, the $d$-dmensional Onofri inequality corresponds to nothing but a natural extension of the optimal Sobolev's inequality. In dimension $d=2$, with $p=2, a=q+1>2$ and $b=2 q$, it has been recently observed in Dolbeault 2011] that

$$
1 \leq \lim _{q \rightarrow \infty} C_{2, q+1} \frac{\left\|\nabla f_{q}\right\|_{L^{2}\left(\mathbb{R}^{2}\right)}^{\frac{q-1}{2 q}}\left\|f_{q}\right\|_{L^{q+1}\left(\mathbb{R}^{2}\right)}^{\frac{q+1}{2 q}}}{\left\|f_{q}\right\|_{\mathrm{L}^{2 q}\left(\mathbb{R}^{2}\right)}}=\frac{e^{\frac{1}{16 \pi} \int_{\mathbb{R}^{2}}|\nabla u|^{2} d x}}{\int_{\mathbb{R}^{2}} e^{u} d \mu_{2}}
$$

if $f_{q}=\left(1+|x|^{2}\right)^{-\frac{1}{q-1}}\left(1+\frac{u}{2 q}\right)$ and $\int_{\mathbb{R}^{2}} u d \mu_{2}=0$. In that sense, Onofri's inequality in dimension $d=2$ replaces Sobolev's inequality in higher dimensions as an endpoint of the family of Gagliardo-Nirenberg inequalities

$$
\|f\|_{\mathrm{L}^{2 q}\left(\mathbb{R}^{d}\right)} \leq \mathrm{C}_{2, q+1}\|\nabla f\|_{\mathrm{L}^{2}\left(\mathbb{R}^{d}\right)}^{\theta}\|f\|_{\mathrm{L}^{q+1}\left(\mathbb{R}^{d}\right)}^{1-\theta}
$$

with $\theta=\frac{q-1}{q} \frac{d}{d+2-q(d-2)}$. In dimension $d \geq 3$, we will see below that (3) can also be seen as an endpoint of (5). 
4 del Pino, M., and Dolbeault, J.

\section{Proof of Theorem 1.1}

Assume that $u \in \mathcal{D}\left(\mathbb{R}^{d}\right)$ is such that $\int_{\mathbb{R}^{d}} u d \mu_{d}=0$ and let

$$
f_{a}:=F_{a}\left(1+\frac{d-1}{d a} u\right)
$$

where $F_{a}$ is defined by

$$
F_{a}(x)=\left(1+|x|^{\frac{d}{d-1}}\right)^{-\frac{d-1}{a-d}} \quad \forall x \in \mathbb{R}^{d} .
$$

From Theorem 1.3, Inequality (5), we know that

$$
1 \leq \lim _{a \rightarrow+\infty} C_{d, a} \frac{\left\|\nabla f_{a}\right\|_{L^{d}\left(\mathbb{R}^{d}\right)}^{\theta}\left\|f_{a}\right\|_{\mathrm{L}^{a}\left(\mathbb{R}^{d}\right)}^{1-\theta}}{\left\|f_{a}\right\|_{\mathrm{L}^{b}\left(\mathbb{R}^{d}\right)}}
$$

if $p=d$. Our goal is to identify the right hand side in terms of $u$. We recall that $b=\frac{d(a-1)}{d-1}$ and $\theta=\frac{a-d}{d(a-1)}$. Using the fact that $F_{a}$ is an optimal function, we can then rewrite (5) with $f=f_{a}$ as

$$
\frac{\int_{\mathbb{R}^{d}}\left|f_{a}\right|^{\frac{d(a-1)}{d-1}} d x}{\int_{\mathbb{R}^{d}}\left|F_{a}\right|^{\frac{d(a-1)}{d-1}} d x} \leq\left(\frac{\int_{\mathbb{R}^{d}}\left|\nabla f_{a}\right|^{d} d x}{\int_{\mathbb{R}^{d}}\left|\nabla F_{a}\right|^{d} d x}\right)^{\frac{a-d}{d(d-1)}} \frac{\int_{\mathbb{R}^{d}}\left|f_{a}\right|^{a} d x}{\int_{\mathbb{R}^{d}}\left|F_{a}\right|^{a} d x}
$$

and observe that:

(i) $\lim _{a \rightarrow+\infty} \int_{\mathbb{R}^{d}}\left|F_{a}\right|^{\frac{d(a-1)}{d-1}} d x=\int_{\mathbb{R}^{d}}\left(1+|x|^{\frac{d}{d-1}}\right)^{-d} d x=\frac{1}{d}\left|\mathbb{S}^{d-1}\right|$ and

$$
\lim _{a \rightarrow+\infty} \int_{\mathbb{R}^{d}}\left|f_{a}\right|^{\frac{d(a-1)}{d-1}} d x=\lim _{a \rightarrow+\infty} \int_{\mathbb{R}^{d}} F_{a}^{\frac{d(a-1)}{d-1}}\left(1+\frac{d-1}{d a} u\right)^{\frac{d(a-1)}{d-1}} d x=\int_{\mathbb{R}^{d}} \frac{e^{u}}{\left(1+|x|^{\frac{d}{d-1}}\right)^{d}} d x,
$$

so that

$$
\lim _{a \rightarrow+\infty} \frac{\int_{\mathbb{R}^{d}}\left|f_{a}\right|^{\frac{d(a-1)}{d-1}} d x}{\int_{\mathbb{R}^{d}}\left|F_{a}\right|^{\frac{d(a-1)}{d-1}} d x}=\int_{\mathbb{R}^{d}} e^{u} d \mu_{d}
$$

(ii) As $a \rightarrow+\infty$,

$$
\int_{\mathbb{R}^{d}}\left|F_{a}\right|^{a} d x \approx \frac{2 a \pi^{d / 2}}{d^{2} \Gamma(d / 2)}, \quad \lim _{a \rightarrow+\infty} \int_{\mathbb{R}^{d}}\left|f_{a}\right|^{a} d x=\infty
$$

and

$$
\lim _{a \rightarrow+\infty} \frac{\int_{\mathbb{R}^{d}}\left|f_{a}\right|^{a} d x}{\int_{\mathbb{R}^{d}}\left|F_{a}\right|^{a} d x}=1
$$

(iii) Finally, as $a \rightarrow+\infty$, we also find that

$$
\left(\frac{\int_{\mathbb{R}^{d}}\left|\nabla f_{a}\right|^{d} d x}{\int_{\mathbb{R}^{d}}\left|\nabla F_{a}\right|^{d} d x}\right)^{\frac{a-d}{d(d-1)}} \approx\left(1+\frac{d(d-1)}{a} \alpha_{d} \int_{\mathbb{R}^{d}} \mathrm{H}_{d}(x, \nabla u) d x\right)^{\frac{a-d}{d(d-1)}} \approx \exp \left(\alpha_{d} \int_{\mathbb{R}^{d}} \mathrm{H}_{d}(x, \nabla u) d x\right) .
$$

Here and above $\ell_{1}(a) \approx \ell_{2}(a)$ means that $\lim _{a \rightarrow+\infty} \ell_{1}(a) / \ell_{2}(a)=1$. Fact (iii) requires some computations which we make explicit next. First of all, we have

$$
\int_{\mathbb{R}^{d}}\left|\nabla F_{a}\right|^{d} d x=\frac{2 d^{d-2} \pi^{d / 2}}{\Gamma(d / 2)} a^{1-d} .
$$

With $X_{a}:=\left(1+\frac{d-1}{d a} u\right) \nabla F_{a}$ and $Y_{a}:=\frac{d-1}{d a} F_{a} \nabla u$, we can write, using the definition of $\mathrm{R}_{d}$, that

$$
\left|\nabla f_{a}\right|^{d}=\left|\nabla F_{a}\right|^{d}\left(1+\frac{d-1}{d a} u\right)^{d}+F_{a}\left|\nabla F_{a}\right|^{d-2} \nabla F_{a} \cdot \nabla\left(1+\frac{d-1}{d a} u\right)^{d}+\mathrm{R}_{d}\left(X_{a}, Y_{a}\right) .
$$

Consider the second term of the right hand side and integrate by parts. A straightforward computation shows that

$$
\int_{\mathbb{R}^{d}} F_{a}\left|\nabla F_{a}\right|^{d-2} \nabla F_{a} \cdot \nabla\left(1+\frac{d-1}{d a} u\right)^{d} d x=-\int_{\mathbb{R}^{d}}\left|\nabla F_{a}\right|^{d}\left(1+\frac{d-1}{d a} u\right)^{d} d x-\int_{\mathbb{R}^{d}} F_{a} \Delta_{d} F_{a}\left(1+\frac{d-1}{d a} u\right)^{d} d x
$$


where $\Delta_{p} F_{a}=\nabla \cdot\left(\left|\nabla F_{a}\right|^{p-2} \nabla F_{a}\right)$ is computed for $p=d$. Collecting terms, we get

$$
\int_{\mathbb{R}^{d}}\left|\nabla f_{a}\right|^{d} d x=-\int_{\mathbb{R}^{d}} F_{a} \Delta_{d} F_{a}\left(1+\frac{d-1}{d a} u\right)^{d} d x+\int_{\mathbb{R}^{d}} \mathrm{R}_{d}\left(X_{a}, Y_{a}\right) d x .
$$

We may next observe that

$$
a \nabla F_{a}(x)=-\frac{d a}{a-d}|x|^{-\frac{d-2}{d-1}} x\left(1+|x|^{\frac{d}{d-1}}\right)_{+}^{-\frac{a-1}{a-d}} \rightarrow-d \frac{|x|^{-\frac{d-2}{d-1}} x}{1+|x|^{\frac{d}{d-1}}} \quad \text { a.e. } \quad \text { as } \quad a \rightarrow+\infty,
$$

while $a \nabla\left(1+\frac{d-1}{d a} u\right)=\frac{d-1}{d} \nabla u$, so that both $X_{a}=\left(1+\frac{d-1}{d a} u\right) \nabla F_{a}$ and $Y_{a}=\frac{d-1}{d a} F_{a} \nabla u$ in $\mathrm{R}_{d}\left(X_{a}, Y_{a}\right)$ are of the order of $1 / a$. By homogeneity, it follows that

$$
a^{d} \mathrm{R}_{d}\left(X_{a}, Y_{a}\right) \rightarrow \mathrm{R}_{d}\left(-\frac{d|x|^{-\frac{d-2}{d-1}}}{1+|x|^{\frac{d}{d-1}}} x, \frac{d-1}{d} \nabla u\right)=\mathrm{H}_{d}(x, \nabla u) \quad \text { as } \quad a \rightarrow+\infty,
$$

by definition of $\mathrm{H}_{d}$. Hence we have established the fact that

$$
\begin{aligned}
& \int_{\mathbb{R}^{d}}\left|\nabla f_{a}\right|^{d} d x=-\int_{\mathbb{R}^{d}} F_{a} \Delta_{d} F_{a}\left(1+\frac{d-1}{d a} u\right)^{d} d x+\int_{\mathbb{R}^{d}} \mathrm{R}_{d}\left(X_{a}, Y_{a}\right) d x \\
&=-\int_{\mathbb{R}^{d}} F_{a} \Delta_{d} F_{a}\left(1+d \frac{d-1}{d a} u+o\left(a^{-1}\right)\right) d x+a^{-d} \int_{\mathbb{R}^{d}} \mathrm{H}_{d}(x, \nabla u) d x
\end{aligned}
$$

Next we can observe that $-\int_{\mathbb{R}^{d}} F_{a} \Delta_{d} F_{a} d x=\int_{\mathbb{R}^{d}}\left|\nabla F_{a}\right|^{d} d x$, while $-\lim _{a \rightarrow+\infty} a^{d-1} F_{a} \Delta_{d} F_{a}=d^{d-1}\left|\mathbb{S}^{d-1}\right| \mu_{d}$, so that

$$
-\int_{\mathbb{R}^{d}} F_{a} \Delta_{d} F_{a} u d x=a^{1-d} d^{d-1}\left|\mathbb{S}^{d-1}\right| \int_{\mathbb{R}^{d}} u d \mu_{d}+o\left(a^{1-d}\right)=o\left(a^{1-d}\right) \quad \text { as } \quad a \rightarrow+\infty
$$

by the assumption that $\int_{\mathbb{R}^{d}} u d \mu_{d}=0$. Altogether, this means that

$$
\left(\frac{\int_{\mathbb{R}^{d}}\left|\nabla f_{a}\right|^{d} d x}{\int_{\mathbb{R}^{d}}\left|\nabla F_{a}\right|^{d} d x}\right)^{\frac{a-d}{d(d-1)}} \approx\left(1+\frac{\int_{\mathbb{R}^{d}} \mathrm{H}_{d}(x, \nabla u) d x}{a^{d} \int_{\mathbb{R}^{d}}\left|\nabla F_{a}\right|^{d} d x}\right)^{\frac{a-d}{d(d-1)}} \approx\left(1+\frac{d(d-1)}{a} \alpha_{d} \int_{\mathbb{R}^{d}} \mathrm{H}_{d}(x, \nabla u) d x\right)^{\frac{a-d}{d(d-1)}}
$$

as $a \rightarrow+\infty$, which concludes the proof of (iii).

Before proving the optimality of the constant $\alpha_{d}$, let us state an intermediate result which of interest in itself. Let us assume that $d \geq 2$ and define $\mathrm{Q}_{d}$ as

$$
\mathrm{Q}_{d}(X, Y):=2 \lim _{\varepsilon \rightarrow 0} \varepsilon^{-2} \mathrm{R}_{d}(X, \varepsilon Y)=\frac{d^{2}}{d t^{2}}|X+t Y|_{\mid t=0}^{d}=d|X|^{d-4}\left[(d-2)(X \cdot Y)^{2}+|X|^{2}|Y|^{2}\right] .
$$

We also define

$$
\mathrm{G}_{d}(x, p):=\mathrm{Q}_{d}\left(-\frac{d|x|^{-\frac{d-2}{d-1}}}{1+|x|^{\frac{d}{d-1}}} x, \frac{d-1}{d} p\right), \quad(x, p) \in \mathbb{R}^{d} \times \mathbb{R}^{d} .
$$

Corollary 2.1. With $\alpha_{d}$ as in Theorem 1.1, we have

$$
\int_{\mathbb{R}^{d}}|v-\bar{v}|^{2} d \mu_{d} \leq \alpha_{d} \int_{\mathbb{R}^{d}} \mathrm{G}_{d}(x, \nabla v) d x \quad \text { with } \quad \bar{v}=\int_{\mathbb{R}^{d}} v d \mu_{d},
$$

for any $v \in \mathrm{L}^{1}\left(\mathbb{R}^{d}, d \mu_{d}\right)$ such that $\nabla v \in \mathrm{L}^{2}\left(\mathbb{R}^{d}, d x\right)$.

This inequality is a Poincaré inequality, which is remarkable. Indeed, if we prove that the optimal constant in (7) is equal to $\alpha_{d}$, then $\alpha_{d}$ is also optimal in Theorem 1.1. Inequality (3). We will see below that this is the case.

Proof of Corollary [2.1. Inequality (7) is a straightforward consequence of (3), written with $u$ replaced by $\varepsilon v$. In the limit $\varepsilon \rightarrow 0$, both sides of the inequality are of order $\varepsilon^{2}$. Details are left to the reader.

To conclude the proof of Theorem 1.1 let us check that there is a nontrivial function $v$ which achieves equality in (7). Since $F_{a}$ is optimal for (3), we can write that

$$
\log \left(\int_{\mathbb{R}^{d}}\left|F_{a}\right|^{\frac{d(a-1)}{d-1}} d x\right)=\log C_{d, a}+\frac{a-d}{d(d-1)} \log \left(\int_{\mathbb{R}^{d}}\left|\nabla F_{a}\right|^{d} d x\right)+\log \left(\int_{\mathbb{R}^{d}}\left|F_{a}\right|^{a} d x\right) .
$$


However, equality also holds true if we replace $F_{a}$ by $F_{a, \varepsilon}$ with $F_{a, \varepsilon}(x):=F_{a}(x+\varepsilon$ e), for an arbitrary given $\mathrm{e} \in \mathbb{S}^{d-1}$, and it is clear that one can differentiate twice with respect to $\varepsilon$ at $\varepsilon=0$. Hence, for any $a>d$, we have

$$
\frac{d(a-1)}{d-1}\left(\frac{d(a-1)}{d-1}-1\right) \frac{\int_{\mathbb{R}^{d}}\left|F_{a}\right|^{\frac{d(a-1)}{d-1}}\left|v_{a}\right|^{2} d x}{\int_{\mathbb{R}^{d}}\left|F_{a}\right|^{\frac{d(a-1)}{d-1}} d x}=\frac{a-d}{d(d-1)} \frac{\int_{\mathbb{R}^{d}} \mathrm{Q}_{d}\left(X_{a}, \frac{d-1}{d} Y_{a}\right) d x}{\int_{\mathbb{R}^{d}}\left|\nabla F_{a}\right|^{d} d x}+a(a-1) \frac{\int_{\mathbb{R}^{d}}\left|F_{a}\right|^{a}\left|v_{a}\right|^{2} d x}{\int_{\mathbb{R}^{d}}\left|F_{a}\right|^{a} d x}
$$

with $X_{a}=\nabla F_{a}, Y_{a}=\frac{d}{d-1} F_{a} \nabla v_{a}$ and $v_{a}:=\mathrm{e} \cdot \nabla \log F_{a}$, that is

$$
v_{a}(x)=-\frac{d}{a-d} \frac{x \cdot \mathrm{e}}{|x|^{\frac{d-2}{d-1}}\left(1+|x|^{\frac{d}{d-1}}\right)}
$$

Hence, if $\phi$ is a radial function, we may notice that $\int_{\mathbb{R}^{d}} \phi v_{a} d x=0$ and

$$
\lim _{a \rightarrow+\infty} a^{2} \int_{\mathbb{R}^{d}} \phi\left|v_{a}\right|^{2} d x=d^{2} \int_{\mathbb{R}^{d}} \phi(x) \frac{|x|^{\frac{2}{d-1}}-2(x \cdot \mathrm{e})^{2}}{\left(1+|x|^{\frac{d}{d-1}}\right)^{2}} d x=d \int_{\mathbb{R}^{d}} \phi(x) \frac{|x|^{\frac{2}{d-1}}}{\left(1+|x|^{\frac{d}{d-1}}\right)^{2}} d x
$$

Since $\int_{\mathbb{R}^{d}}\left|F_{a}\right|^{\frac{d(a-1)}{d-1}} d x=o\left(\int_{\mathbb{R}^{d}}\left|F_{a}\right|^{a} d x\right)$, the last term in (8) is negligible compared to the other ones. Passing to the limit as $a \rightarrow+\infty$, with $v:=\lim _{a \rightarrow+\infty} a v_{a}$, we find that $v$ is given by (4) and

$$
\left(\frac{d}{d-1}\right)^{2} \int_{\mathbb{R}^{d}}|v|^{2} d \mu_{d}=\alpha_{d} \int_{\mathbb{R}^{d}} \mathrm{Q}_{d}\left(-\frac{d|x|^{-\frac{d-2}{d-1}}}{1+|x|^{\frac{d}{d-1}}} x, \frac{d-1}{d} Y\right) d x
$$

where $Y:=\frac{d-1}{d} \nabla v$ and where we have used the fact that

$$
d(d-1) \alpha_{d} \lim _{a \rightarrow+\infty} a^{d} \int_{\mathbb{R}^{d}}\left|\nabla F_{a}\right|^{d} d x=1
$$

Since the function $\mathrm{Q}_{d}$ is quadratic, we obtain that

$$
\left(\frac{d}{d-1}\right)^{2} \int_{\mathbb{R}^{d}}|v|^{2} d \mu_{d}=\alpha_{d} \int_{\mathbb{R}^{d}} \mathrm{G}_{d}\left(x, \frac{d}{d-1} \nabla v\right) d x=\alpha_{d}\left(\frac{d}{d-1}\right)^{2} \int_{\mathbb{R}^{d}} \mathrm{G}_{d}(x, \nabla v) d x,
$$

which corresponds precisely to equality in (7) since $v$ given by (4) is such that $\bar{v}=0$.

Equality in (3) is achieved by constants. The optimality of $\alpha_{d}$ amounts to establish that in the inequality

$$
\mathcal{Q}_{d}[u] \geq \frac{1}{\alpha_{d}}
$$

equality can be achieved along a minimizing sequence. Notice that

$$
\mathcal{Q}_{d}[u]=\frac{\int_{\mathbb{R}^{d}} \mathrm{H}_{d}(x, \nabla u) d x}{\log \left(\int_{\mathbb{R}^{d}} e^{u} d \mu_{d}\right)} \quad \text { if } \quad \int_{\mathbb{R}^{d}} u d \mu_{d}=0
$$

The reader is invited to check that $\lim _{\varepsilon \rightarrow 0} \mathcal{Q}_{d}[\varepsilon v]=\frac{1}{\alpha_{d}}$. In dimension $d=2, v$ is an eigenfunction associated to the eigenvalue problem: $-\Delta v=\lambda_{1} v \mu_{2}$, corresponding to the lowest positive eigenvalue, $\lambda_{1}$. The generalization to higher dimensions is given by (4). Notice that the function $v$ is an eigenfunction of the linear form associated to $\mathrm{G}_{d}$, in the space $L^{2}\left(\mathbb{R}^{d}, d \mu_{d}\right)$. This concludes the proof of Theorem 1.1

Whether there are non-trivial optimal functions, that is, whether there exists a non-constant function $u$ such that $\mathcal{Q}_{d}[u]=\frac{1}{\alpha_{d}}$, is an open question. At least the proof of Theorem 1.1 shows that there is a loss of compactness in the sense that the limit of $\varepsilon v$, i.e. 0 , is not an admissible function for $\mathcal{Q}_{d}$.

Acknowlegments. J.D. has been supported by the projects CBDif and EVOL of the French National Research Agency (ANR). M.D. has been supported by grants Fondecyt 1110181 and Fondo Basal CMM. Both authors are participating to the MathAmSud network NAPDE. 


\section{References}

Thierry Aubin. Problèmes isopérimétriques et espaces de Sobolev. J. Differential Geometry, 11(4):573-598, 1976. ISSN 0022-040X.

Thierry Aubin. Meilleures constantes dans le théorème d'inclusion de Sobolev et un théorème de Fredholm non linéaire pour la transformation conforme de la courbure scalaire. J. Funct. Anal., 32(2):148-174, 1979. ISSN 0022-1236. doi: 10.1016/0022-1236(79)90052-1. URL https://proxy.bu.dauphine.fr:443/http/dx.doi.org/10.1016/0022-1236(79)90052-1.

William Beckner. Sharp Sobolev inequalities on the sphere and the Moser-Trudinger inequality. Ann. of Math. (2), 138(1):213-242, 1993. ISSN 0003-486X. doi: $10.2307 / 2946638$. URL http://dx.doi.org/10.2307/2946638.

GA Bliss. An integral inequality. Journal of the London Mathematical Society, 1(1):40, 1930. ISSN 0024-6107.

E. Carlen and M. Loss. Competing symmetries, the logarithmic HLS inequality and Onofri's inequality on $\mathbb{S}^{n}$. Geom. Funct. Anal., 2(1):90-104, 1992. ISSN 1016-443X. doi: 10.1007/BF01895706. URL http://dx.doi.org/10.1007/BF01895706

Dario Cordero-Erausquin, Wilfrid Gangbo, and Christian Houdré. Inequalities for generalized entropy and optimal transportation. In Recent advances in the theory and applications of mass transport, volume 353 of Contemp. Math., pages 73-94. Amer. Math. Soc., Providence, RI, 2004.

Manuel Del Pino and Jean Dolbeault. Nonlinear diffusions and optimal constants in Sobolev type inequalities: asymptotic behaviour of equations involving the $p$-Laplacian. C. R. Math. Acad. Sci. Paris, 334(5):365-370, 2002a. ISSN 1631-073X.

Manuel Del Pino and Jean Dolbeault. Best constants for Gagliardo-Nirenberg inequalities and applications to nonlinear diffusions. J. Math. Pures Appl. (9), 81(9):847-875, 2002b. ISSN 0021-7824.

Manuel Del Pino and Jean Dolbeault. The optimal Euclidean $L^{p}$-Sobolev logarithmic inequality. J. Funct. Anal., 197(1):151-161, 2003. ISSN 0022-1236.

Manuel Del Pino, Jean Dolbeault, and Ivan Gentil. Nonlinear diffusions, hypercontractivity and the optimal $L^{p}$-Euclidean logarithmic Sobolev inequality. J. Math. Anal. Appl., 293(2):375-388, 2004. ISSN 0022-247X.

Jean Dolbeault. Sobolev and Hardy-Littlewood-Sobolev inequalities: duality and fast diffusion. To appear in Math. Res. Lett., 2011.

Jean Dolbeault, Maria J. Esteban, and Gabriella Tarantello. The role of Onofri type inequalities in the symmetry properties of extremals for Caffarelli-Kohn-Nirenberg inequalities, in two space dimensions. Ann. Sc. Norm. Super. Pisa Cl. Sci. (5), 7(2):313-341, 2008. ISSN 0391-173X.

Ivan Gentil. The general optimal $L^{p}$-Euclidean logarithmic Sobolev inequality by Hamilton-Jacobi equations. J. Funct. Anal., 202(2):591-599, 2003. ISSN 0022-1236. doi: 10.1016/S0022-1236(03)00047-8. URL https://proxy.bu.dauphine.fr:443/http/dx.doi.org/10.1016/S0022-1236(03)00047-8.

Alessandro Ghigi. On the Moser-Onofri and Prékopa-Leindler inequalities. Collect. Math., 56(2):143-156, 2005. ISSN 0010-0757.

Bernd Kawohl and Marcello Lucia. Best constants in some exponential Sobolev inequalities. Indiana Univ. Math. J., 57(4):1907-1927, 2008. ISSN 0022-2518. doi: 10.1512/iumj.2008.57.3307. URL http://dx.doi.org/10.1512/iumj.2008.57.3307.

M Kim. Linearised Moser-Trudinger inequality. Bull. Austral. Math. Soc., 62:445-457, 2000.

J. Moser. A sharp form of an inequality by N. Trudinger. Indiana Univ. Math. J., 20:1077-1092, 1970/71. ISSN $0022-2518$.

E. Onofri. On the positivity of the effective action in a theory of random surfaces. Comm. Math. Phys., 86(3): 321-326, 1982. ISSN 0010-3616. 
8 del Pino, M., and Dolbeault, J.

Gerald Rosen. Minimum value for $c$ in the Sobolev inequality $\left\|\phi^{3}\right\| \leq c\|\nabla \phi\|^{3}$. SIAM J. Appl. Math., 21:30-32, 1971. ISSN 0036-1399.

Giorgio Talenti. Best constant in Sobolev inequality. Ann. Mat. Pura Appl. (4), 110:353-372, 1976. ISSN 0003-4622. 